\title{
A DIRECT BOUNDARY ELEMENT METHOD FOR SIGNORINI PROBLEMS
}

\author{
HOUDE HAN
}

\begin{abstract}
In this paper, a Signorini problem is reduced to a variational inequality on the boundary, and a direct boundary element method is presented for its solution. Furthermore, error estimates for the approximate solutions of Signorini problems are given. In addition, we show that the Signorini problem may be formulated as a saddle-point problem on the boundary.
\end{abstract}

\section{INTRODUCTION}

The Signorini problems are a class of very important variational inequalities, which arise in many practical problems such as the elasticity with unilateral conditions $[17,5]$, the fluid mechanics problems in media with semipermeable boundaries [4, 7], the electropaint process [1], etc. In the literature, there are many authors who have studied the problems, established the existence and uniqueness, and obtained the regularity results for Signorini problems (see Brézis [3] and Friedman [6]). Furthermore, the numerical solution of the Signorini problem by the finite element method has been discussed [7, 8]. We know that the solution of the Signorini problem satisfies a linear partial differential equation in the domain, even when the problem is nonlinear. Hence, it is natural and advantageous to apply the boundary element method to Signorini problems. An indirect boundary element method for solving Signorini problems has been presented in [9].

In this paper, we will present a direct boundary element method for solving the same problem. First, using the Calderón projector for the traces and the normal derivative of the solution of the Signorini problem, this latter is reduced to a variational inequality on the boundary $\Gamma$ based on a direct boundary element method. The resulting variational inequality on the boundary involves both weakly singular and hypersingular boundary integral operators. The bilinear form arising in this boundary inequality is continuous and coercive on suitable subspaces of the Sobolev space $H^{1 / 2}(\Gamma) \times H^{-/ 12}(\Gamma)$. This leads to

Received February 11, 1988; revised November 29, 1988.

1980 Mathematics Subject Classification (1985 Revision). Primary 65N45, 49A29.

Key words and phrases. Signorini problem, variational inequality, boundary finite element method, saddle-point problem.

This work is supported in part by the National Natural Science Foundation of China, and by the U.S.N.S.F., grant INT-8517582. 
existence and uniqueness for the solution of the boundary variational inequality. Furthermore, the Signorini problem can also be formulated as a saddlepoint problem involving only boundary integral operators on $\Gamma$, which finally we can solve with a uniform boundary element Galerkin method and obtain quasioptimal error estimates for the Galerkin error.

Let $\Omega$ be a bounded domain in $\mathbf{R}^{2}$ with a smooth boundary $\Gamma$. Suppose $\Gamma=\Gamma_{0} \cup \Gamma_{1}$ (as shown in Figure 1), with $\Gamma_{0} \neq \varnothing$. We define

$$
\begin{aligned}
& \stackrel{*}{H}^{1}(\Omega)=\left\{u \in H^{1}(\Omega),\left.u\right|_{\Gamma_{0}}=0\right\}, \\
& a(u, v)=\int_{\Omega} \nabla u \cdot \nabla v d x, \\
& L(v)=\int_{\Omega} f v d x+\int_{\Gamma_{1}} g v d s \\
& K=\left\{v \in \stackrel{*}{H}^{1}(\Omega), v \geq 0 \text { a.e. on } \Gamma_{1}\right\} \\
& \widetilde{K}=\{\operatorname{trace} \text { of } v \text { on } \Gamma, v \in K\} \\
& \stackrel{*}{H}^{1 / 2}(\Gamma)=\left\{\operatorname{trace} \text { of } v \text { on } \Gamma, v \in \stackrel{H}{H}^{1}(\Omega)\right\} \\
& \stackrel{*}{H}^{-/ 12}(\Gamma)=\left\{\mu \in H^{-1 / 2}(\Gamma), \int_{\Gamma} \mu d s=0\right\}, \\
& V=\stackrel{*}{H}^{1 / 2}(\Gamma) \times \stackrel{*}{H}^{-1 / 2}(\Gamma), \quad \text { with norm }\|(v, \mu)\|_{V}^{2}=\|v\|_{1 / 2, \Gamma}^{2}+\|\mu\|_{-1 / 2, \Gamma}^{2},
\end{aligned}
$$

where $H^{m}(\Omega)$ and $H^{\alpha}(\Gamma)$ denote the usual Sobolev spaces, $m, \alpha$ are two real numbers (see [15]), and $f \in H^{-1}(\Omega)$ and $g \in H^{-1 / 2}(\Gamma)$ are given functions. We know that $K$ is a closed convex set in $\stackrel{*}{H}^{1}(\Omega)$.

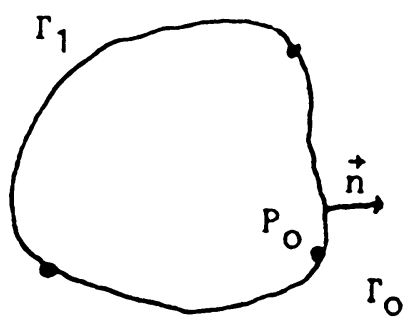

FIGURE 1

We consider the following variational inequality:

find $u \in K$ such that

$$
a(u, v-u) \geq L(v-u) \forall v \in K \text {. }
$$

Problem (1.1) is a model for Signorini problems. The following theorems are known [8].

Theorem 1.1. The variational inequality (1.1) has a unique solution. 
Theorem 1.2. The solution of problem (1.1) is characterized by

$$
\begin{array}{ll}
-\Delta u=f & \text { a.e. in } \Omega, \\
u=0 & \text { a.e. on } \Gamma_{0}, \\
u \geq 0, \partial u / \partial n \geq g & \text { a.e. on } \Gamma_{1}, \\
u(\partial u / \partial n-g)=0 & \text { a.e. on } \Gamma_{1} .
\end{array}
$$

Next, let $u_{0}$ be the solution of the following boundary value problem:

$$
\begin{aligned}
-\Delta u_{0}=f & \text { in } \Omega, \\
u_{0}=0 & \text { on } \Gamma .
\end{aligned}
$$

Hence, we have by the first Green's formula with $u \in H^{*}(\Omega)$ and $f \in L^{2}(\Omega)$

$$
\begin{aligned}
-a\left(u_{0}, v+u_{0}-u\right)= & -\int_{\Omega} f\left(v+u_{0}-u\right) d x \\
& -\int_{\Gamma_{1}} \frac{\partial u_{0}}{\partial n}\left(v+u_{0}-u\right) d s \quad \forall v \in K .
\end{aligned}
$$

From (1.1) and (1.4) we obtain with $u, u_{0} \in K$,

$$
a\left(u-u_{0}, v-\left(u-u_{0}\right)\right) \geq \int_{\Gamma_{1}}\left(g-\frac{\partial u_{0}}{\partial n}\right)\left(v-\left(u-u_{0}\right)\right) d s \quad \forall v \in K .
$$

Let $w=u-u_{0}$ and $g^{*}=g-\partial u_{0} / \partial n$; then $w$ solves the following problem:

Find $w \in K$ such that

$$
a(w, v-w) \geq \int_{\Gamma_{1}} g^{*}(v-w) d s \quad \forall v \in K .
$$

Therefore, without loss of generality, we may assume that $f \equiv 0$. In this case, problem (1.1) is reduced to

Find $u \in K$ such that

$$
a(u, v-u) \geq \int_{\Gamma_{1}} g(v-u) d s \quad \forall v \in K .
$$

\section{AN EQUIVALENT BOUNDARY VARIATIONAL INEQUALITY FOR PROBLEM $(1.1)^{*}$}

Suppose that $u$ is the solution of problem $(1.1)^{*}$; then in the domain $\Omega$, $\Delta u=0$. Let $\lambda=\partial u /\left.\partial n\right|_{\Gamma} \in H^{-1 / 2}(\Gamma)$. By Green's formula we obtain

$$
u(x)=\int_{\Gamma} \frac{\partial G(x, y)}{\partial n_{y}} u(y) d s_{y}-\int_{\Gamma} G(x, y) \lambda(y) d s_{y} \quad \forall x \in \Omega,
$$

where $G(x, y)=\frac{1}{2 \pi} \log |x-y|, x \neq y ; n_{y}$ denotes the outward unit normal to $\Gamma=\partial \Omega$ at $y \in \Gamma$. By the properties of the single-layer and double-layer potentials, we obtain the first relationship between $\lambda$ and $\left.u\right|_{\Gamma}$ :

$$
\frac{1}{2} u(x)=\int_{\Gamma} \frac{\partial G(x, y)}{\partial n_{y}} u(y) d s_{y}-\int_{\Gamma} G(x, y) \lambda(y) d s_{y} \quad \forall x \in \Gamma .
$$


Furthermore, using the behavior of the derivative of the single-layer and doublelayer potentials $[10,12,16]$, we get

$$
\frac{1}{2} \lambda(x)=\int_{\Gamma} \frac{\partial^{2} G(x, y)}{\partial n_{x} \partial n_{y}} u(y) d s_{y}-\int_{\Gamma} \frac{\partial G(x, y)}{\partial n_{x}} \lambda(y) d s_{y} \quad \forall x \in \Gamma,
$$

where

$$
\int_{\Gamma} \frac{\partial^{2} G(x, y)}{\partial n_{x} \partial n_{y}} u(y) d s_{y}=\frac{d}{d s_{x}} \int_{\Gamma} G(x, y) \frac{d u(y)}{d s_{y}} d s_{y} \quad \forall x \in \Gamma .
$$

Multiplying (2.2) by a function $\mu \in \stackrel{*}{H}^{-1 / 2}(\Gamma)$, we get

$$
\begin{gathered}
-\frac{1}{2} \int_{\Gamma} u(x) \mu(x) d s_{x}+\int_{\Gamma} \int_{\Gamma} \frac{\partial G(x, y)}{\partial n_{y}} u(y) \mu(x) d s_{y} d s_{x} \\
-\int_{\Gamma} \int_{\Gamma} G(x, y) \lambda(y) \mu(x) d s_{y} d s_{x}=0 .
\end{gathered}
$$

Let

$$
\begin{gathered}
a_{0}(\mu, \lambda)=-\int_{\Gamma} \int_{\Gamma} G(x, y) \lambda(y) \mu(x) d s_{y} d s_{x}, \\
b(\mu, u)=\frac{1}{2} \int_{\Gamma} u(x) \mu(x) d s_{x}-\int_{\Gamma} \int_{\Gamma} \frac{\partial G(x, y)}{\partial n_{y}} u(y) \mu(x) d s_{y} d s_{x}
\end{gathered}
$$

then equation (2.4) can be rewritten as follows:

$$
-b(\mu, u)+a_{0}(\mu, \lambda)=0 \quad \forall \mu \in \stackrel{*}{H}^{-1 / 2}(\Gamma) .
$$

For any $v \in \stackrel{*}{H}^{1}(\Omega)$, we have, for $u$ satisfying $\Delta u=0$ in $\Omega$,

$$
a(u, v)=\int_{\Omega} \nabla u \cdot \nabla v d x=\int_{\Gamma} \lambda v d s .
$$

Inserting (2.3) into (2.5) and integrating by parts, we obtain

$$
\begin{aligned}
a(u, v)= & -\int_{\Gamma} \int_{\Gamma} G(x, y) \frac{d u(y)}{d s_{y}} \frac{d v(x)}{d s_{x}} d s_{y} d s_{x} \\
& -\int_{\Gamma} \int_{\Gamma} \frac{\partial G(x, y)}{\partial n_{x}} \lambda(y) v(x) d s_{y} d s_{x}+\frac{1}{2} \int_{\Gamma} \lambda v d s \\
= & a_{0}\left(\frac{d u}{d s}, \frac{d v}{d s}\right)+b(\lambda, v) .
\end{aligned}
$$

Hence, the variational inequality $(1.1)^{*}$ is reduced to the following boundary variational inequality:

Find $(u, \lambda) \in \widetilde{K} \times \stackrel{*}{H}^{-1 / 2}(\Gamma)$ such that

$$
\begin{aligned}
& a_{0}\left(\frac{d u}{d s}, \frac{d v}{d s}-\frac{d u}{d s}\right)+b(\lambda, v-u) \geq \int_{\Gamma_{1}} g(v-u) d s \quad \forall v \in \widetilde{K}, \\
& -b(\mu, u)+a_{0}(\mu, \lambda)=0 \quad \forall \mu \in \stackrel{H}{H}^{-1 / 2}(\Gamma)
\end{aligned}
$$


or,

$$
\begin{aligned}
& \text { find }(u, \lambda) \in \widetilde{K} \times \stackrel{*}{H}^{-1 / 2}(\Gamma) \text { such that } \\
& A(u, \lambda ; v-u, \mu) \geq \int_{\Gamma_{1}} g(v-u) d s \quad \forall v \in \widetilde{K}, \mu \in \stackrel{*}{H}^{-1 / 2}(\Gamma),
\end{aligned}
$$

where

$$
A(u, \lambda ; v, \mu)=a_{0}\left(\frac{d u}{d s}, \frac{d v}{d s}\right)+b(\lambda, v)-b(\mu, u)+a_{0}(\mu, \lambda)
$$

is a bilinear form on $V \times V$.

We now prove the following lemmas:

Lemma 2.1. There is a constant $\alpha_{0}>0$ such that

$$
\|d v / d s\|_{-1 / 2, \Gamma} \geq \alpha_{0}\|v\|_{1 / 2, \Gamma} \quad \forall v \in \stackrel{*}{H}^{1 / 2}(\Gamma) .
$$

Proof. Let $\theta=2 \pi s / L$, where $L$ denotes the length of the boundary $\Gamma$ and $s$ denotes the length of the segment from the point $P_{0} \in \Gamma$ to a point $p \in \Gamma$ along the boundary $\Gamma$. Hence, we only need to prove $(2.8)$ when $\Gamma$ is the circle with radius 1 . On the other hand, we know that the space $\stackrel{*}{C}^{\infty}(\Omega)=\left\{v \in C^{\infty}(\Omega)\right.$, support of $\left.v \subset \bar{\Omega} \backslash \Gamma_{0}\right\}$ is dense in $H^{*}(\Omega)$. If we can prove (2.8) for any $v \in \stackrel{*}{C}^{\infty}(\Omega)$, then $(2.8)$ holds for $v \in \stackrel{H}{H}^{1 / 2}(\Gamma)$.

Suppose $\Gamma$ is the circle with radius 1 . For any $v \in \stackrel{C}{C}^{\infty}(\Omega)$, on $\Gamma$ we have

$$
v=\frac{a_{0}}{2}+\sum_{n=1}^{\infty}\left(a_{n} \cos n \theta+b_{n} \sin n \theta\right),
$$

where

$$
\begin{gathered}
a_{n}=\frac{1}{\pi} \int_{0}^{2 \pi} v(\theta) \cos n \theta d \theta, \quad n=0,1,2, \ldots, \\
b_{n}=\frac{1}{\pi} \int_{0}^{2 \pi} v(\theta) \sin n \theta d \theta, \quad n=1,2, \ldots
\end{gathered}
$$

We know that $[14$, p. 21$]$

$$
\|v\|_{1 / 2, \Gamma}^{2}=\frac{a_{0}^{2}}{2}+\sum_{n=1}^{\infty}\left(1+n^{2}\right)^{1 / 2}\left(a_{n}^{2}+b_{n}^{2}\right) .
$$

Furthermore, we have

$$
\begin{aligned}
& \frac{d v}{d \theta}=\sum_{n=1}^{\infty}\left(n b_{n} \cos n \theta-n a_{n} \sin n \theta\right) \\
& \left\|\frac{d v}{d \theta}\right\|_{-1 / 2, \Gamma}^{2}=\sum_{n=1}^{\infty}\left(1+n^{2}\right)^{-1 / 2}\left(n^{2} b_{n}^{2}+n^{2} a_{n}^{2}\right) \\
& \geq \frac{1}{2} \sum_{n=1}^{\infty}\left(1+n^{2}\right)^{1 / 2}\left(a_{n}^{2}+b_{n}^{2}\right) .
\end{aligned}
$$


On the other hand, we know that

$$
a_{0}=\frac{1}{\pi} \int_{0}^{2 \pi} v(\theta) d \theta=-\frac{1}{\pi} \int_{0}^{2 \pi} \frac{d v(\theta)}{d \theta} \theta d \theta
$$

By $\left.v\right|_{\Gamma_{0}}=0$, that is, $v(\theta)=0,-\delta_{1} \leq \theta \leq \delta_{2} \quad\left(\delta_{1}, \delta_{2}>0\right)$, there is a function $\phi(\theta) \in C^{\infty}(\Gamma)$ such that $\left.\phi\right|_{\Gamma_{1}}=1, \phi(\theta)=0$, for $|\theta| \leq \frac{1}{2} \min \left(\delta_{1}, \delta_{2}\right)$. Hence, we get

$$
a_{0}=-\frac{1}{\pi} \int_{0}^{2 \pi} \frac{d v}{d \theta} \theta \phi(\theta) d \theta
$$

where $\theta \phi(\theta) \in C^{\infty}(\Gamma)$. Furthermore, we obtain

$$
\begin{aligned}
\left|a_{0}\right| & \leq \frac{1}{\pi}\|\theta \phi(\theta)\|_{1 / 2, \Gamma}\left\|\frac{d v}{d \theta}\right\|_{-1 / 2, \Gamma} \\
& \equiv C_{0}\left\|\frac{d v}{d \theta}\right\|_{-1 / 2, \Gamma}, \quad C_{0}=\frac{1}{\pi}\|\theta \phi(\theta)\|_{1 / 2, \Gamma}>0, \\
& \left\|\frac{d v}{d \theta}\right\|_{-1 / 2, \Gamma}^{2} \geq \frac{1}{C_{0}^{2}}\left|a_{0}\right|^{2} .
\end{aligned}
$$

Combining (2.9), (2.10), and (2.11), we obtain the inequality (2.8) with $\alpha_{0}=$ $\min \left(1 / \sqrt{2}, 1 / \sqrt{2} C_{0}\right)$.

Lemma 2.2. $A(u, \lambda ; v, \mu)$ is a bounded bilinear form on $V \times V$; that is, there exists a constant $M>0$ such that

$$
|A(u, \lambda ; v, \mu)| \leq M\|(u, \lambda)\|_{v}\|(v, \mu)\|_{v} \quad \forall(u, \lambda),(v, \mu) \in V .
$$

Furthermore, there is a constant $\beta>0$ such that

$$
A(v, \mu ; v, \mu) \geq \beta\|(v, \mu)\|_{v}^{2} \quad \forall(v, \mu) \in V .
$$

Proof. It is straightforward to check that $A(u, \lambda ; v, \mu)$ is a bounded bilinear form on $V \times V$ (see [11]). We now prove inequality (2.13). We have

$$
A(v, \mu ; v, \mu)=a_{0}\left(\frac{d v}{d s}, \frac{d v}{d s}\right)+a_{0}(\mu, \mu) .
$$

We recall (see [13]) that the bounded bilinear form $a_{0}(\lambda, \mu)$ is $\stackrel{*}{H}^{-1 / 2}(\Gamma)$ elliptic, i.e., there exists a positive constant $\beta_{0}$ such that

$$
a_{0}(\mu, \mu) \geq \beta_{0}\|\mu\|_{-1 / 2, \Gamma}^{2} \quad \forall \mu \in \stackrel{H}{H}^{-1 / 2}(\Gamma) .
$$

Hence,

$$
a_{0}\left(\frac{d v}{d s}, \frac{d v}{d s}\right) \geq \beta_{0}\left\|\frac{d v}{d s}\right\|_{-1 / 2, \Gamma}^{2} \quad \forall v \in H^{1 / 2}(\Gamma) .
$$

Combining (2.14), (2.15), (2.16), and (2.8), we get inequality (2.13) with $\beta=$ $\min \left(\beta_{0}, \alpha_{0} \beta_{0}\right)>0$. 
Lemma 2.3. Suppose $u \in \stackrel{*}{H}^{1 / 2}(\Gamma) \cap H^{1 / 2+\alpha}(\Gamma)$ and $\lambda \in \stackrel{*}{H}^{-1 / 2}(\Gamma) \cap H^{-1 / 2+\alpha}(\Gamma)$ $(0 \leq \alpha \leq 1)$; then there exists a constant $M_{\alpha}>0$ such that

$$
\begin{aligned}
|A(u, \lambda ; v, \mu)| \leq & M_{\alpha}\left(\|u\|_{1 / 2+\alpha, \Gamma}^{2}+\|\lambda\|_{-1 / 2+\alpha, \Gamma}^{2}\right)^{1 / 2} \\
& \cdot\left(\|v\|_{1 / 2-\alpha, \Gamma}^{2}+\|\mu\|_{1 / 2-\alpha, \Gamma}^{2}\right)^{1 / 2} \quad \forall(v, \mu) \in V .
\end{aligned}
$$

Proof. We consider the operators

$$
K_{0}: H^{r}(\Gamma) \rightarrow H^{r+1}(\Gamma), \quad-\frac{3}{2} \leq r,
$$

where $K_{0} \phi=-\int_{\Gamma} G(x, y) \phi(y) d s_{y} \quad \forall \phi(y) \in H^{r}(\Gamma)$, and

$$
K_{1}: H^{r}(\Gamma) \rightarrow H^{r}(\Gamma), \quad-\frac{1}{2} \leq r,
$$

where $K_{1} \phi=\int_{\Gamma}\left(\partial G(x, y) / \partial n_{y}\right) \phi(y) d s_{y} \quad \forall \phi \in H^{r}(\Gamma)$. We know (see [10]) that $K_{0}$ and $K_{1}$ are bounded operators. Let $\langle v, \mu\rangle=\int_{\Gamma} v \mu d s$; then we obtain

$$
\begin{aligned}
A(u, \lambda ; v, \mu)= & \left\langle K_{0}\left(\frac{d u}{d s}\right), \frac{d v}{d s}\right\rangle+\left\langle K_{0}(\lambda), \mu\right\rangle \\
& +\frac{1}{2}\langle v, \lambda\rangle-\frac{1}{2}\langle u, \mu\rangle-\left\langle K_{1} v, \lambda\right\rangle+\left\langle K_{1} u, \mu\right\rangle .
\end{aligned}
$$

Furthermore, we have for $0 \leq \alpha \leq 1$

$$
\begin{aligned}
\left|\left\langle K_{0}\left(\frac{d u}{d s}\right), \frac{d v}{d s}\right\rangle\right| & \leq\left\|K_{0}\left(\frac{d u}{d s}\right)\right\|_{1 / 2+\alpha, \Gamma}\left\|\frac{d v}{d s}\right\|_{-1 / 2-\alpha, \Gamma} \\
& \leq\left\|K_{0}\right\|\left\|\frac{d u}{d s}\right\|_{-1 / 2-\alpha, \Gamma}\|v\|_{1 / 2-\alpha, \Gamma} \\
& \leq\left\|K_{0}\right\|\|u\|_{1 / 2+\alpha, \Gamma}\|v\|_{1 / 2-\alpha, \Gamma}, \\
|\langle v, \lambda\rangle| & \leq\|\lambda\|_{-1 / 2+\alpha, \Gamma}\|v\|_{1 / 2-\alpha, \Gamma} \\
\left|\left\langle K_{1} v, \lambda\right\rangle\right| & \leq\|\lambda\|_{-1 / 2+\alpha, \Gamma}\left\|K_{1} v\right\|_{1 / 2-\alpha, \Gamma} \\
& \leq C_{\alpha}\|\lambda\|_{-1 / 2+\alpha, \Gamma}\|v\|_{1 / 2-\alpha, \Gamma}
\end{aligned}
$$

where

$$
C_{\alpha}=\sup _{v \in H^{1 / 2-a}(\Gamma)} \frac{\left\|K_{1} v\right\|_{1 / 2-\alpha, \Gamma}}{\|v\|_{1 / 2-\alpha, \Gamma}} .
$$

Similarly, we get

$$
\begin{aligned}
\left|\left\langle K_{0} \lambda, \mu\right\rangle\right| & \leq\left\|K_{0}\right\|\|\lambda\|_{-1 / 2+\alpha, \Gamma}\|\mu\|_{-1 / 2-\alpha, \Gamma}, \\
|\langle u, \mu\rangle| & \leq\|u\|_{1 / 2+\alpha, \Gamma}\|\mu\|_{-1 / 2-\alpha, \Gamma}, \\
\left|\left\langle K_{1} u, \mu\right\rangle\right| & \leq \widetilde{C}_{\alpha}\|\mu\|_{1 / 2+\alpha, \Gamma}\|\mu\|_{-1 / 2-\alpha, \Gamma},
\end{aligned}
$$

where $\widetilde{C}_{\alpha}=\sup _{u \in H^{1 / 2+\alpha}(\Gamma)}\left\|K_{1}(u)\right\|_{1 / 2+\alpha, \Gamma} /\|u\|_{1 / 2+\alpha, \Gamma}$. Then inequality $(2.17)$ follows immediately.

By Lemma 2.2, an application of Theorem 2.1 in [8, Chapter I] yields the following result. 
Theorem 2.1. Suppose that $g \in H^{-1 / 2}\left(\Gamma_{1}\right)$; then the variational inequality (2.7) (or (2.6)) has a unique solution $(u, \lambda) \in \widetilde{K} \times H^{*}-1 / 2(\Gamma)$.

Suppose $(u, \lambda) \in \widetilde{K} \times \stackrel{*}{H}^{-1 / 2}(\Gamma)$ is the solution of (2.7). By formula (2.1), we obtain the function $u(x) \in \stackrel{*}{H}^{1}(\Omega)$ and $\partial u / \partial n=\lambda$; then, for any $v \in \stackrel{*}{H}^{1}(\Omega)$ and $\mu \in \stackrel{*}{H}^{-1 / 2}(\Gamma)$, we have with $\Delta u=0$ in $\Omega$ :

$$
a(u, v-u)=\int_{\Omega} \nabla u \cdot \nabla v d x=\int_{\Gamma} \lambda(v-u) d s=A(u, \lambda ; v-u, \mu) .
$$

Hence, $u(x) \in \stackrel{H}{H}^{1}(\Omega)$ is the solution of the original problem $(1.1)^{*}$. This means that the boundary variational inequality $(2.7)$ is equivalent to problem $(1.1)^{*}$. Furthermore, we have the following

Theorem 2.2. The variational inequality (2.6) is equivalent to the following saddlepoint problem:

$$
\begin{aligned}
& \text { Find }(u, \lambda) \in \widetilde{K} \times \stackrel{*}{H}^{-1 / 2}(\Gamma) \text { such that } \\
& L(u, \mu) \leq L(u, \lambda) \leq L(v, \lambda) \quad \forall v \in \widetilde{K}, \mu \in \stackrel{*}{H}^{-1 / 2}(\Gamma),
\end{aligned}
$$

where

$$
L(v, \mu)=\frac{1}{2} a_{0}\left(\frac{d v}{d s}, \frac{d v}{d s}\right)+b(\mu, v)-\frac{1}{2} a_{0}(\mu, \mu)-\int_{\Gamma_{1}} g v d s .
$$

Proof. Suppose that $(u, \lambda) \in \widetilde{K} \times \stackrel{*}{H}^{-1 / 2}(\Gamma)$ is the solution of (2.18). Then, for any $\mu \in \stackrel{*}{H}^{-1 / 2}(\Gamma)$ and any real number $\varepsilon, \lambda+\varepsilon \mu \in \stackrel{*}{H}^{-1 / 2}(\Gamma)$; we have

$$
L(u, \lambda+\varepsilon \mu) \leq L(u, \lambda),
$$

that is,

$$
\varepsilon\left[b(\mu, u)-a_{0}(\mu, \lambda)\right]-\frac{\varepsilon^{2}}{2} a_{0}(\mu, \mu) \leq 0 .
$$

Since $\varepsilon$ is an arbitrary constant, we obtain

$$
-b(\mu, u)+a_{0}(\mu, \lambda)=0 \quad \forall \mu \in \stackrel{*}{H}^{-1 / 2}(\Gamma) .
$$

On the other hand, for any $u, v \in \widetilde{K}$, we know that $u+t(v-u) \in \widetilde{K} \quad(0 \leq$ $t \leq 1)$; then $\widetilde{K}$ is convex, and we get

$$
L(u, \lambda) \leq L(u+t(v-u), \lambda) \quad \forall v \in \tilde{K},
$$

that is,

$$
\begin{gathered}
t\left[a_{0}\left(\frac{d u}{d s}, \frac{d v}{d s}-\frac{d u}{d s}\right)+b(\lambda, v-u)-\int_{\Gamma_{1}} g(v-u) d s\right] \\
+\frac{t^{2}}{2} a_{0}\left(\frac{d(v-u)}{d s}, \frac{d(v-u)}{d s}\right) \geq 0 .
\end{gathered}
$$


Since $1 \geq t \geq 0$ is an arbitrary constant, we get

$$
a_{0}\left(\frac{d u}{d s}, \frac{d(v-u)}{d s}\right)+b(\lambda, v-u) \geq \int_{\Gamma_{1}} g(v-u) d s \quad \forall v \in \widetilde{K} .
$$

This means that $(u, \lambda) \in \widetilde{K} \times \stackrel{*}{H}^{-1 / 2}(\Gamma)$ is the solution of (2.6). Each of the above steps is reversible, and we conclude that if $(u, \lambda)$ is a solution of $(2.6)$, then it is a solution of $(2.18)$.

\section{THE DISCRETE APPROXIMATION OF}

THE BOUNDARY VARIATIONAL INEQUALITY (2.7)

Suppose that $S_{h_{1}}$ and $S_{h_{2}}$ are two finite-dimensional subspaces of $\stackrel{*}{H}^{1 / 2}(\Gamma)$ and $\stackrel{*}{H}^{-1 / 2}(\Gamma)$, respectively. Let $\widetilde{K}_{h_{1}}=\left\{v_{h}, v_{h} \in S_{h_{1}} \cup \widetilde{K}\right\}$. Moreover, we assume that $\widetilde{K}_{h_{1}}$ is a closed convex nonempty subset of $S_{h_{1}}$.

Now we consider the discrete problem

Find $\left(u_{h}, \lambda_{h}\right) \in \widetilde{K}_{h_{1}} \times S_{h_{2}}$ such that

$$
A\left(u_{h}, \lambda_{h} ; v_{h}-u_{h}, \mu_{h}\right) \geq \int_{\Gamma_{1}} g\left(v_{h}-u_{h}\right) d s \quad \forall v_{h} \in \widetilde{K}_{h_{1}}, \mu_{h} \in S_{h_{2}} .
$$

It is straightforward to prove:

Theorem 3.1. The problem (3.1) has a unique solution $\left(u_{h}, \lambda_{h}\right) \in \widetilde{K}_{h_{1}} \times S_{h_{2}}$. Furthermore, we obtain the error estimate stated in the following theorem.

Theorem 3.2. Suppose that the solution of $(2.7),(u, \lambda)$, satisfies $u \in \widetilde{K} \cup$ $H^{1 / 2+\alpha}(\Gamma), \lambda \in H^{* 1 / 2}(\Gamma) \cup H^{-1 / 2+\alpha}(\Gamma)$, and $g \in H^{-1 / 2+\alpha}(\Gamma)$, where $0 \leq \alpha \leq 1$; then the following error estimate holds:

$$
\begin{aligned}
\left\|\left(u-u_{h}, \lambda-\lambda_{h}\right)\right\|_{v}^{2} \leq C_{\alpha} & \left\{\inf _{v_{h} \in \widetilde{K}_{h_{1}}}\left[\left\|u-v_{h}\right\|_{1 / 2, \Gamma}^{2}+\left\|u-v_{h}\right\|_{1 / 2-\alpha, \Gamma}\right]\right. \\
& \left.+\inf _{\mu_{h} \in S_{h_{2}}}\left[\left\|\lambda-\mu_{h}\right\|_{-1 / 2, \Gamma}^{2}+\left\|\lambda-\mu_{h}\right\|_{-1 / 2-\alpha, \Gamma}\right]\right\},
\end{aligned}
$$

where $C_{\alpha}$ is a constant independent of $h_{1}$ and $h_{2}$.

Proof. Taking $v=u_{h}$ and $\mu=\lambda_{h}-\lambda$ in (2.7), we have

$$
-A\left(u, \lambda ; u_{h}-u, \lambda_{h}-\lambda\right) \leq-\int_{\Gamma_{1}} g\left(u_{h}-u\right) d s .
$$

Similarly, we get

$$
\text { (3.4) }-A\left(u_{h}, \lambda_{h} ; v_{h}-u_{h}, \mu_{h}-\lambda_{h}\right) \leq-\int_{\Gamma_{1}} g\left(v_{h}-u_{h}\right) d s \quad \forall v_{h} \in \widetilde{K}_{h_{1}}, \mu_{h} \in S_{h_{2}} \text {. }
$$


On the other hand,

$$
\begin{aligned}
& \begin{array}{l}
\left\|\left(u-u_{h}, \lambda-\lambda_{h}\right)\right\|_{V}^{2} \leq \frac{1}{\beta} A\left(u-u_{h}, \lambda-\lambda_{h} ; u-u_{h}, \lambda-\lambda_{h}\right) \\
=\frac{1}{\beta}\left\{A\left(u-u_{h}, \lambda-\lambda_{h} ; u-v_{h}, \lambda-\mu_{h}\right)\right. \\
\quad+A\left(u, \lambda ; v_{h}-u, \mu_{h}-\lambda\right) \\
\quad-A\left(u_{h}, \lambda_{h} ; v_{h}-u_{h}, \mu_{h}-\lambda_{h}\right) \\
\left.\quad+A\left(u, \lambda ; u_{h}-u, \lambda_{h}-\lambda\right)\right\} \\
\quad+A\left(u, \lambda ; v_{h}-u, \mu_{h}-\lambda\right) \\
\left.\quad-\int_{\Gamma_{1}} g\left(v_{h}-u\right) d s\right\} \quad \forall v_{h} \in \widetilde{K}_{h_{1}}, \mu_{h} \in S_{h_{2}} .
\end{array}
\end{aligned}
$$

Combining (3.5), (2.12), and (2.17), we obtain

$$
\begin{aligned}
&\left\|\left(u-u_{h}, \lambda-\lambda_{h}\right)\right\|_{V}^{2} \leq \frac{1}{\beta}\{ M\left\|\left(u-u_{h}, \lambda-\lambda_{h}\right)\right\|_{V} \|\left(u-v_{h}, \lambda-\mu_{h} \|_{V}\right. \\
&+ M_{\alpha}\left(\|u\|_{1 / 2+\alpha, \Gamma}^{2}+\|\lambda\|_{-1 / 2+\alpha, \Gamma}^{2}\right)^{1 / 2} \\
& \cdot\left(\left\|u-v_{h}\right\|_{1 / 2-\alpha, \Gamma}^{2}+\left\|\lambda-\mu_{h}\right\|_{1 / 2-\alpha, \Gamma}^{2}\right)^{1 / 2} \\
&\left.\quad+\|g\|_{-1 / 2+\alpha, \Gamma}\left\|u-v_{h}\right\|_{1 / 2-\alpha, \alpha, \Gamma}\right\} .
\end{aligned}
$$

Then the error estimate (3.2) follows immediately.

Assume that the boundary $\Gamma$ of $\Omega$ is represented as

$$
\chi_{1}=\chi_{1}(S), \quad \chi_{2}=\chi_{2}(S), \quad 0 \leq S \leq L,
$$

and $\chi_{j}(0)=\chi_{j}(L), j=1,2$. Furthermore, $\Gamma$ is divided into segments $\{T\}$ by the points $\chi^{i}=\left(\chi_{1}\left(S_{i}\right), \chi_{2}\left(S_{i}\right)\right), i=1,2, \ldots, N_{1}$, that include the two endpoints of $\Gamma_{0}$, with $S_{1}=0, S_{N_{1}+1}=L$; we define

$$
h_{1}=\max _{1 \leq i \leq N_{1}}\left|S_{i+1}-S_{i}\right| \text {. }
$$

This partition of $\Gamma$ is denoted by $\mathscr{T}_{h_{1}}$. Let

$$
\begin{aligned}
& S_{h_{1}}=\left\{v_{h} \in C^{0}(\Gamma),\left.v_{h}\right|_{T}\right. \text { is a linear function } \\
& \left.\qquad \forall T \in \mathscr{T}_{h_{1}} \text { and }\left.v_{h}\right|_{\Gamma_{0}}=0\right\}, \\
& \tilde{K}_{h_{1}}=\left\{v_{h} \in S_{h_{1}}, v_{h} \geq 0 \text { on } \Gamma_{1}\right\} .
\end{aligned}
$$

Similarly, we have another partition $\mathscr{T}_{h_{2}}$. Let

$$
S_{h_{2}}=\left\{\left.\mu_{h}\right|_{T} \text { is a constant } \forall T \in \mathscr{T}_{h_{2}} \text { and } \int_{\Gamma} \mu_{h} d s=0\right\} .
$$

Obviously, $\widetilde{K}_{h_{1}}$ is a closed convex subset of $S_{h_{1}}$, and it is nonempty. The subspaces $S_{h_{1}}$ and $S_{h_{2}}$ are two regular finite element spaces in the sense of 
Babuška and Aziz [2] that satisfy the following approximation property:

$$
\begin{gathered}
\inf _{v_{h} \in \widetilde{K}_{h_{1}}}\left\{\left\|u-v_{h}\right\|_{1 / 2, \Gamma}^{2}+\left\|u-v_{h}\right\|_{1 / 2-\alpha, \Gamma}\right\} \\
\leq C_{\alpha}^{1} h_{1}^{2 \alpha}\left[\|u\|_{1 / 2+\alpha, \Gamma}^{2}+\|u\|_{1 / 2+\alpha, \Gamma}\right], \\
\inf _{\mu_{h} \in S_{h_{2}}}\left\{\left\|\lambda-\mu_{h}\right\|_{-1 / 2, \Gamma}^{2}+\left\|\lambda-\mu_{h}\right\|_{-1 / 2-\alpha, \Gamma}\right\} \\
\leq C_{\alpha}^{2} h_{2}^{2 \alpha}\left[\|\lambda\|_{-1 / 2+\alpha, \Gamma}^{2}+\|\lambda\|_{-1 / 2+\alpha, \Gamma}\right] .
\end{gathered}
$$

Combining (3.9), (3.10), and (3.2), we obtain

Corollary. Suppose that the subspaces $S_{h_{1}}$ and $S_{h_{2}}$ are given by (3.7) and (3.8); moreover, let the solution $(u, \lambda)$ satisfy the assumptions of Theorem 3.2. Then the following error estimate holds:

$$
\begin{aligned}
\left\|\left(u-u_{h}, \lambda-\lambda_{h}\right)\right\|_{v}^{2} \leq \widetilde{C}_{\alpha}\{ & h_{1}^{2 \alpha}\left[\|u\|_{1 / 2+\alpha, \Gamma}^{2}+\|u\|_{1 / 2+\alpha, \Gamma}\right] \\
& \left.+h_{2}^{2 \alpha}\left[\|\lambda\|_{-1 / 2+\alpha, \Gamma}^{2}+\|\lambda\|_{-1 / 2+\alpha}\right]\right\},
\end{aligned}
$$

where $\widetilde{C}_{\alpha}$ is a constant independent of $h_{1}$ and $h_{2}$.

\section{THE SOLUTION OF THE BOUNDARY VARIATIONAL INEQUALITY (3.1)}

In this section, we will present an approach for solving problem (3.1). Let $a_{1}(u, v)=a_{0}(d u / d s, d v / d s)$; then problem (3.1) can be rewritten as follows:

Find $\left(u_{h}, \lambda_{h}\right) \in \widetilde{K}_{h_{1}} \times S_{h_{2}}$ such that

$$
\begin{aligned}
& a_{1}\left(u_{h}, v_{h}-u_{h}\right)+b\left(\lambda_{h}, v_{h}-u_{h}\right) \geq \int_{\Gamma_{1}} g\left(v_{h}-u_{h}\right) d s, \quad v_{h} \in \widetilde{K}_{h_{1}}, \\
& -b\left(\mu_{h}, u_{h}\right)+a_{0}\left(\mu_{h}, \lambda_{h}\right)=0, \quad \forall \mu_{h} \in S_{h_{2}} .
\end{aligned}
$$

As a first step, for any given $u \in H^{1 / 2}(\Gamma)$, we solve the following problem:

Find $\lambda_{h}(u) \in S_{h_{2}}$ such that

$$
a_{0}\left(\mu_{h}, \lambda_{h}\right)=b\left(\mu_{h}, u\right) \quad \forall \mu_{h} \in S_{h_{2}} .
$$

Problem (4.1) has a unique solution $\lambda_{h}(u)$, and

$$
\left\|\lambda_{h}(u)\right\|_{-/ 12, \Gamma} \leq\|b\|\|u\|_{1 / 2, \Gamma} / \beta_{0} .
$$

Let $\mu_{i}(x), i=1,2, \ldots, N_{2}$, denote a basis for the space $S_{h_{2}}$, and

$$
\lambda_{h}(u)=\sum_{i=1}^{N_{2}} \lambda_{i}(u) \mu_{i}(x)=\lambda^{T} \cdot \boldsymbol{\mu}(x),
$$

where $\lambda^{T}=\left(\lambda_{1}(u), \lambda_{2}(u), \ldots, \lambda_{N_{2}}(u)\right)$ and $\boldsymbol{\mu}(x)^{T}=\left(\mu_{1}(x), \mu_{2}(x), \ldots\right.$, $\left.\mu_{N_{2}}(x)\right)$. From (4.1), we obtain

$$
\mathbf{G} \lambda=\mathbf{F}(u)
$$


where $\mathbf{G}=\left(a_{0}\left(\mu_{i}, \mu_{j}\right)\right)_{N_{2} \times N_{2}}$ and

$$
\mathbf{F}(u)^{T}=\left(b\left(\mu_{1}, u\right), b\left(\mu_{2}, u\right), \ldots, b\left(\mu_{N_{2}}, u\right)\right) .
$$

$\mathbf{G}$ is a symmetric and positive definite matrix. Hence we get

$$
\lambda=\mathbf{G}^{-1} \mathbf{F}(u)
$$

and $\lambda_{h}(u)=\lambda^{T} \cdot \boldsymbol{\mu}(x)$. Let $A_{h_{2}}(u, v)=a_{1}(u, v)+b\left(\lambda_{h}(u), v\right)$; then $A_{h_{2}}(u, v)$ is a bilinear form on $H^{1 / 2}(\Gamma) \times H^{1 / 2}(\Gamma)$. Furthermore, we have:

Lemma 4.1. (i) $A_{h_{2}}(u, v)$ is a bounded bilinear form on $H^{1 / 2}(\Gamma) \times H^{1 / 2}(\Gamma)$; that is, there exists a constant $M>0$ independent of $h_{2}$ such that

$$
\left|A_{h_{2}}(u, v)\right| \leq M\|u\|_{1 / 2, \Gamma}\|v\|_{1 / 2, \Gamma} \quad \forall u, v \in H^{1 / 2}(\Gamma) .
$$

(ii) $A_{h_{2}}(u, v)$ is symmetric.

(iii) $A_{h_{2}}(u, v)$ is $\stackrel{*}{H}^{1 / 2}(\Gamma)$-elliptic uniformly for $h_{2}$; that is, there exists a constant $\gamma>0$ such that

$$
A_{h_{2}}(v, v) \geq \gamma\|v\|_{1 / 2, \Gamma}^{2} \quad \forall v \in \stackrel{*}{H}^{1 / 2}(\Gamma) .
$$

Proof. (i) We have

$$
\begin{aligned}
\left|A_{h_{2}}(u, v)\right| & =\left|a_{1}(u, v)+b\left(\lambda_{h}(u), v\right)\right| \\
& \leq\left\|a_{1}\right\|\|u\|_{1 / 2, \Gamma}\|v\|_{1 / 2, \Gamma}+\|b\|\left\|\lambda_{h}(u)\right\|_{-1 / 2, \Gamma}\|v\|_{1 / 2, \Gamma} \\
& \leq\left\{\left\|a_{1}\right\|+\|b\|^{2} / \beta_{0}\right\}\|u\|_{1 / 2, \Gamma}\|v\|_{1 / 2, \Gamma} .
\end{aligned}
$$

The inequality (4.4) holds with $M=\left\|a_{1}\right\|+\|b\|^{2} / \beta_{0}$.

(ii) From (4.1), we get

$$
\begin{aligned}
& a_{0}\left(\lambda_{h}(v), \lambda_{h}(u)\right)=b\left(\lambda_{h}(v), u\right), \\
& a_{0}\left(\lambda_{h}(u), \lambda_{h}(v)\right)=b\left(\lambda_{h}(u), v\right) .
\end{aligned}
$$

By symmetry of $a_{0}(\lambda, \mu)$, we obtain $b\left(\lambda_{h}(v), u\right)=b\left(\lambda_{h}(u), v\right)$. Hence,

$$
A_{h_{2}}(u, v)=a_{1}(u, v)+b\left(\lambda_{h}(u), v\right)=a_{1}(v, u)+b\left(\lambda_{h}(v), u\right)=A_{h_{2}}(v, u) \text {. }
$$

(iii) We have

$$
\begin{aligned}
A_{h_{2}}(v, v) & =a_{1}(v, v)+b\left(\lambda_{h}(v), v\right) \\
& =a_{1}(v, v)+a_{0}\left(\lambda_{h}(v), \lambda_{h}(v)\right) \\
& =A\left(v, \lambda_{h}(v) ; v, \lambda_{h}(v)\right) \geq \beta\|v\|_{1 / 2, \Gamma}^{2} \quad \forall v \in \stackrel{*}{H}^{1 / 2}(\Gamma) .
\end{aligned}
$$

In the second step we reduce the boundary variational inequality $(3.1)^{*}$ to:

$$
\text { Find } u_{h} \in \widetilde{K}_{h_{1}} \text { such that }
$$

$$
A_{h_{2}}\left(u_{h}, v_{h}-u_{h}\right) \geq \int_{\Gamma_{1}} g\left(v_{h}-u_{h}\right) d s \quad \forall v_{h} \in \widetilde{K}_{h_{1}} .
$$


Let

$$
J(u)=\frac{1}{2} A_{h_{2}}(u, u)-\int_{\Gamma_{1}} g u d s .
$$

By Lemma 4.1 we obtain [8, p. 10]:

Theorem 4.1. The boundary variational problem (4.6) is equivalent to the following minimization problem on $\widetilde{K}_{h_{1}}$ :

$$
J\left(u_{h}\right)=\min _{v_{h} \in \widetilde{K}_{h_{1}}} J\left(v_{h}\right)
$$

A method for solving problem (4.7) can be found in [7]. After solving (4.7), we can get $\lambda\left(u_{h}\right)$ from problem (4.1); then formula (2.1) gives the solution of the original problem.

\section{ACKNOWLEDGMENT}

My thanks are due to Professor R. B. Kellogg for valuable discussions.

\section{BIBLIOGRAPHY}

1. J. M. Aitchison, A. A. Lacey, and M. Shillor, A model for an electropaint process, IMA J. Appl. Math. 33 (1984), 17-31.

2. I. Babuška and A. K. Aziz, Survey lectures on the mathematical foundations of the finite element method, The Mathematical Foundations of the Finite Element Method with Applications to Partial Differential Equations (A. K. Aziz, ed.), Academic Press, New York, 1972, pp. 5-359.

3. H. Brézis, Problèmes unilatéraux, J. Math. Pures Appl. 51 (1972), 1-168.

4. G. Duvaut and J.-L. Lions, Les inéquations en mécanique et en physique, Dunod, Paris, 1972.

5. G. Fichera, Problemi elastostatici con vincoli unilaterali: il problema di Signorini con ambigue condizioni al contorno, Mem. Accad. Naz. Lincei (8) 7 (1963/64), 91-140.

6. A. Friedman, Variational principles and free-boundary problems, Wiley, New York, 1982.

7. R. Glowinski, Numerical methods for nonlinear variational problems, Springer-Verlag, New York, 1984.

8. R. Glowinski, J.-L. Lions, and R. Trémolières, Numerical analysis of variational inequalities, North-Holland, Amsterdam, 1981.

9. H. Han, The boundary finite element methods for Signorini problems, Lecture Notes in Math., vol. 1297, Springer-Verlag, Berlin and Heidelberg, 1987, pp. 38-49.

10. _ The boundary integro-differential equations of elliptic boundary value problems and their numerical solutions, Sci. Sinica Ser. A 29 (1988), 1153-1165.

11. A A new class of variational formulations for the coupling of finite and boundary element methods (to appear).

12. G. C. Hsiao and W. Wendland, A finite element method for some integral equations of the first kind, J. Math. Anal. Appl. 58 (1977), 447-481.

13. M. N. Le Roux, Méthode d'éléments finis pour la résolution numérique de problèmes exterieurs en dimension 2, RAIRO Anal. Numér. 11 (1977), 27-60.

14. J.-L. Lions, Optimal control of systems governed by partial differential equations, SpringerVerlag, Berlin and Heidelberg, 1971.

15. J.-L. Lions and E. Magenes, Non-homogeneous boundary value problems and applications. I, Springer-Verlag, Berlin, 1972. 
16. J. C. Nedelec, Integral equations with nonintegrable kernels, Integral Equations Operator Theory 5 (1982), 562-572.

17. A. Signorini, Sopra alcune questioni di elastostatica, Atti della Società Italiana per il Progresso della Scienza, 1933.

Department of Applied Mathematics, Tsinghua University, Beijing, People's Republic OF CHINA

Current address: Department of Mathematics, University of Maryland, College Park, Maryland 20742 